\title{
Distribuição sazonal de Culex (Culex) eduardoi Casal \& Garcia (Diptera, Culicidae) em criadouros antrópicos introduzidos em mata residual degradada, área urbana de Curitiba, Paraná, Brasil ${ }^{1}$
}

\author{
Magda Clara Vieira da Costa Ribeiro ${ }^{2}$ \\ Ana Cristina Tissot ${ }^{3}$ \\ Mário Antônio Navarro da Silva ${ }^{3}$
}

\begin{abstract}
Seazonal distribution of Culex (Culex) eduardoi Casal \& Garcia (Diptera, Culicidae) in artificial receptacles in disturbed patch of forest degraded in urban area of Curitiba, Paraná, Brazil. The utilization of artificial receptacles to oviposition by mosquitoes in forest environment may indicate a sinantropic tendency or behaviour. Our data revealed that tires were as the most acceptable breeding for Culex (Culex) eduardoi Casal \& Garcia 1968. The population density of this species was higher and summer seasons.

KEY WORDS. Culicidae, Culex (Culex) eduardoi, tire
\end{abstract}

A utilização de recipientes artificiais pelos culicídeos em ambiente de mata pode evidenciar tendência sinantrópica, já que em condições naturais utilizam outro local de oviposição aos já estabelecidos em seu ecótopo. ForATTINI et al. (1997) relatam que mosquitos apresentam uma mudança comportamental cada vez maior frente a oferta de artefatos antropogênicos e até mesmo adaptação a esse meio modificado. Os produtos gerados pela ação antrópica com capacidade de retenção de água têm sido observados como potenciais criadouros na proliferação de mosquitos em áreas urbana por LOPES et al. (1993), CHAHAD \& LOZOVEI (1994), SILVA \& MENEZES (1996), ALMiRÓN \& BREWER (1996), ForATtini et al. (1998a, b).

A ocorrência de Culex (Culex) eduardoi Casal \& Garcia, 1968 é relatada no Estado do Paraná por SILVA \& LOZOVEI (1996) no município de Curitiba e LOPES (1996, 1997) em área rural do Norte do Paraná, sendo suas formas imaturas encontradas principalmente em recipientes artificiais.

Os habitats das formas imaturas de Culicidae têm merecido importante destaque em estudos ecológicos e epidemiológicos, já que representam o papel de adaptabilidade como resposta à pressão seletiva antrópica a qual caracteriza-se, entre outros aspectos, pelo grande número de recipientes, fruto da moderna tecnologia dos descartáveis (FORATTINI 1998).

1) Contribuição número 1235 do Departamento de Zoologia, Universidade Federal do Paraná.

2) Programa de Pós Graduação em Biologia Molecular e Celular, Fundação Oswaldo Cruz. E-mail: aedes@tecpar.br

3) Departamento de Zoologia da UFPR, Caixa Postal 19020, 81531-980, Curitiba, Paraná. E-mail: mnavarro@bio.ufpr.br 
O objetivo desse trabalho foi avaliar a distribuição sazonal de imaturos de Culex (Culex) eduardoi em recipientes antropogênicos, inseridos em mata degradada, área urbana de Curitiba.

\section{MATERIAL E MÉTODOS}

A área de estudo localizada em perímetro urbano da cidade de Curitiba, Estado do Paraná, Brasil ( $25^{\circ} 25^{\prime} 04 \mathrm{~S}$ e $49^{\circ} 14^{\prime} 30 \mathrm{~W}, 945 \mathrm{~m}$ de altitude), compreende mata residual alterada no campus da Universidade Federal do Paraná, onde encontra-se elementos de campo como Poaceae, Apiaceae, vegetação de brejo localizada nas porções de maior depressão do terreno, com poucos indivíduos arbóreos representados por Verbenaceae e Asteraceae. A composição arbustiva compõem-se de espécies de Anacardiaceae, Bignoniaceae, Myrcinaceae, além de Araucária angustifolia (Bert.). Com relação ao aspecto climático, durante o período que decorreu o estudo, a média máxima anual da temperatura foi de $22^{\circ} \mathrm{C}$ e mínima de $13^{\circ} \mathrm{C}$ com precipitação pluviométrica de $550 \mathrm{~mm}$ e média anual de umidade relativa do ar igual a $78 \%$ para o município de Curitiba (Sistema Meteorológico do Paraná - SIMEPAR).

O período de investigação decorreu de março de 1999 a março de 2000 , sendo as amostragens realizadas mensalmente.

Dentro da mata, foram introduzidos três tipos de recipientes em três blocos e com as seguintes características: a) vaso de cerâmica na cor marrom; b) vaso de plástico na cor preta, ambos com abertura superior de $15 \mathrm{~cm}$ e altura de $13,5 \mathrm{~cm}$; c) pneu de motocicleta secionado transversalmente no sentido de rolagem. Todos os recipientes foram instalados em posição vertical e amarrados ao tronco de uma árvore a uma altura de aproximadamente $1,5 \mathrm{~m}$ do solo e, em seguida, preenchidos com $1400 \mathrm{ml}$ de água destilada, capacidade máxima para todos os recipientes (Fig. 1).

A água dos criadouros era retirada e passada através de peneira com malha de $200 \mu \mathrm{m}$ de diâmetro, sendo devolvida em seguida e, quando necessário, reposta com água destilada. Com relação aos pneus, utilizou-se o método de sifonagem para o esgotamento da água (FORATTINI 1962).

Em cada inspeção mensal foram considerados criadouros observados somente os que estavam aptos a serem colonizados, ou seja, com água. Sendo, portanto, feita reposição de água quando necessário.

No laboratório, parte das larvas foi transferida para recipientes individuais, visando a obtenção de exúvias de larvas, pupas e adultos. Todas as pupas coletadas foram criadas para obtenção de adultos. O restante do material foi sacrificado e acondicionado em álcool a $80 \%$.

Exemplares de Culex (Culex) eduardoi foram depositados na coleção de Entomologia Pe. Jesus Santiago Moure, Departamento de Zoologia, Universidade Federal do Paraná (DZUP).

Os parâmetros ambientais foram obtidos de planilhas meteorológicas da Estação $n^{\circ} 83842$ do SIMEPAR, localizada no Centro Politécnico, Universidade Federal do Paraná, distante aproximadamente $50 \mathrm{~m}$ do ponto de coleta.

Para análise dos resultados, utilizou-se correlação linear simples, médias aritméticas e percentuais dos números absolutos de imaturos coletados (ZAR 1984). 

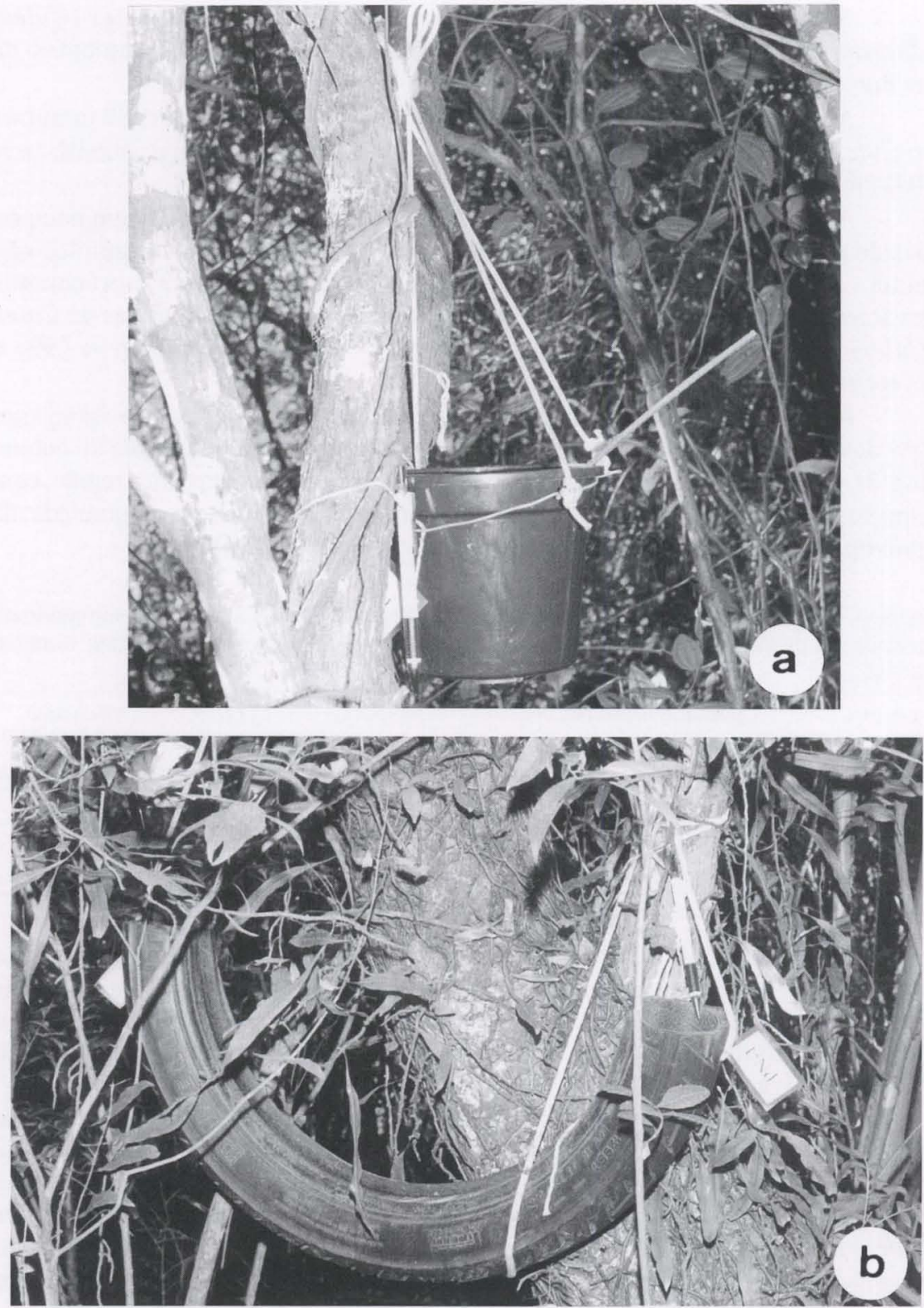

Fig. 1. Recipientes antropogênicos instalados em mata degradada, área urbana, março de 1999 a março de 2000 , Curitiba, Paraná. 


\section{RESULTADOS E DISCUSSÃO}

$\mathrm{Na}$ área de estudo foram encontrados apenas imaturos de Culex (Culex) eduardoi explorando recipientes do tipo pneu e plástico. Os recipientes de cerâmica não receberam oviposições.

Ao final dos treze meses de investigações foram coletados 1959 imaturos de Culex (Culex) eduardoi sendo 79,37\% $(\mathrm{n}=1555)$ em pneus e $20,62 \%(\mathrm{n}=$ 404) em recipientes plásticos (Fig. 2).

A primeira ocorrência de imaturos nos recipientes testados foi em pneu no mês de abril, onde apenas uma larva foi coletada. Nos três meses seguintes não foram coletados imaturos, somente em agosto os recipientes foram procurados novamente para oviposição. Observou-se os maiores números de larvas de Culex (Culex) eduardoi nos meses de novembro $(\mathrm{n}=297)$, dezembro $(\mathrm{n}=199)$ e fevereiro $(n=151)$.

A ocorrência desta espécie foi restrita praticamente à primavera e verão com 97\% dos imaturos coletados, sendo reduzida no inverno (3\%) e inexistente no outono (Fig. 3). O outono e inverno foram marcados, como é característico para a região, com temperatura mínima inferior a $12^{\circ} \mathrm{C}$ e precipitação pluviométrica acumulada de aproximadamente $37 \%$ do somatório para a primavera e verão (Tab. I).

Tabela I. Média estacional e desvio padrão da temperatura máxima e mínima e somatória da precipitação pluviométrica, durante o período de março de 1999 à março de 2000, Curitiba, Paraná.

\begin{tabular}{lccc}
\hline Estações & Temperatura máxima $\left({ }^{\circ} \mathrm{C}\right)$ & Temperatura mínima $\left({ }^{\circ} \mathrm{C}\right)$ & $\begin{array}{c}\text { Precipitação } \\
\text { pluviométrica }(\mathrm{mm})\end{array}$ \\
\cline { 2 - 3 } & Média \pm Desvio padrão & Média \pm Desvio padrão & \\
\hline Verão & $25,7 \pm 0,85$ & $17,0 \pm 0,90$ & 550,00 \\
Outono & $20,2 \pm 2,60$ & $11,1 \pm 2,32$ & 205,80 \\
Inverno & $20,6 \pm 2,19$ & $9,4 \pm 0,84$ & 149,60 \\
Primavera & $22,7 \pm 2,20$ & $13,0 \pm 2,23$ & 409,40 \\
\hline
\end{tabular}

Embora durante a primavera e verão tenha sido observado aumento no número de-imaturos, estações as quais foram registradas as temperaturas mais elevadas para o período de estudo (Tab. I), na análise de correlação entre o número mensal de imaturos e média da temperatura máxima e mínima embora positiva não foi significativa, sendo de 0,29 e 0,18 , respectivamente.

Os recipientes de plástico foram colonizados pela primeira vez na estação da primavera, onde atingiu $16 \%$ das larvas, contribuindo dessa forma com $80 \%$ de imaturos do total coletado neste tipo de criadouro. Dos recipientes positivos para a presença de imaturos, o pneu mostrou-se mais visitado para oviposição de Culex (Culex) eduardoi contribuindo com o total de 172 e os de plástico com 117 do total de espécimens coletados. Dentre as 60 investigações realizadas, aproximadamente $38 \%(n=23)$ foram positivas para pneus e cerca $6 \%(n=4)$ para recipientes plásticos.

A densidade populacional observada para Culex (Culex) eduardoi nos diferentes tipos de recipientes considerados neste trabalho, revela que maior preferência foi para pneus. Como nas estações da primavera e verão foi verificada a 

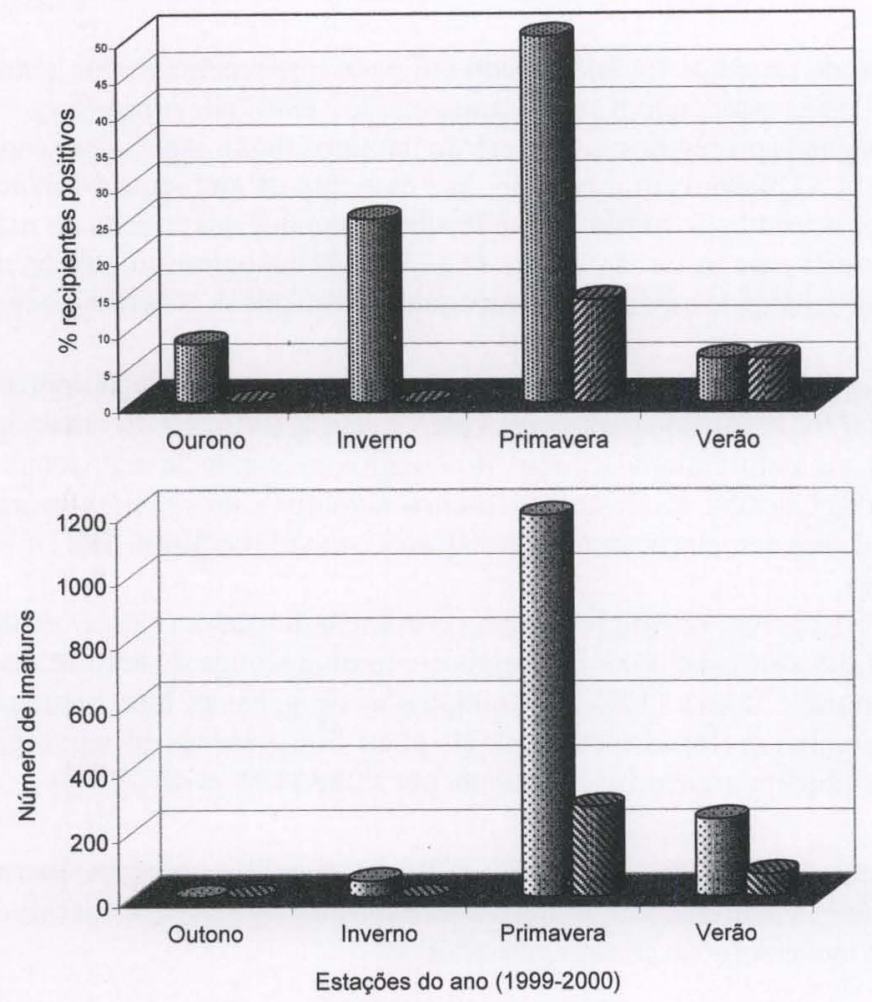

Figs 2-3. (2) Números de imaturos de Culex (Culex) eduardoi coletados nos recipientes artificiais e (3) percentual estacional de recipientes positivos introduzidos em mata degradada, área urbana de Curitiba no período de março de 1999 a março de 2000.

presença desta espécie nos criadouros de plásticos, sugere-se que, possivelmente a oviposição neste tipo de recipiente seja de caráter oportunista, já que os meses de setembro a março coincidem com época de maior número de imaturos desse culicíneo. Resultado similar ao observado por LOPES (1997) em mata ciliar.

Sendo importantes locais de procriação de algumas espécies em área urbana, inclusive as quais normalmente desenvolvem-se em ocos de árvores (BAUMGARTNER 1988), a utilização de pneus e seu elevado potencial como criadouros de mosquitos foi estudado por EASTON (1994), LOPES et al. (1993), GETTMAN \& HALL (1989), NASCI (1988), BERRY et al. (1988) e BEIER et al. (1983).

Não houve homogeneidade quanto à colonização por Culex (Culex) eduardoi nos pneus instalados. O maior número de oviposições foi realizada em pneu que encontrava-se em local mais sombreado próximo de vegetação mais fechada. Possivelmente, uma maior oferta de matéria vegetal aliada a fatores abióticos locais, pode ter influenciado nesse resultado.

O ambiente influindo na oviposição por espécies de Culicidae é mencionado por Silva \& Lozovei (1996) para Culex (Culex) quinquefasciatus Say, 1823, onde 
o maior número de imaturos foi encontrado em pneu que recebia maior sombreamento. NASCI (1988) estudando o comportamento de Aedes triseriatus (Say, 1823) reporta que, em pneus protegidos, o número de imaturos dessa espécie era superior à área descoberta. $\mathrm{O}$ mesmo autor conclui, que esse tipo de ambiente é favorecido devido ao maior acúmulo de folhas, o que resulta em maior quantidade de matéria orgânica disponível para as larvas. BEIER et al. (1983) encontraram diferenças na composição e na densidade específica de mosquitos entre pneus cobertos e expostos por vegetação.

A utilização de recipientes artificiais como locais de oviposição por Culex (Culex) eduardoi foi verificado também por SILVA \& LOZOVEI (1996) em recipientes de cerâmica, cimento-amianto e pneu introduzidos em capão de mata localizado em área urbana de Curitiba. Esses autores mencionam que Culex (Culex) eduardoi pode ser coletada em associação com Culex (Culex) acharistus Root, 1927 e Culex quinquefasciatus.

LOPES \& LOZOVEI (1995) relatam a ocorrência de Culex (Culex) eduardoi em coletas realizadas em criadouros artificiais e naturais ao longo do leito de ribeirão ao Norte do Paraná e LOPES (1997) em recipientes de plástico, lata, bambu num pequeno percentual e em elevada densidade em pneu. Sua presença em caixa d'água localizada em ambiente urbano foi assinalada por FORATTINI et al. (1997).

AGRADECIMENTOS. A Randal Lopes Barreira e a Eduardo Fumio Kuwabara pelo apoio no trabalho de campo. A Marcelo Duarte pela revisão do manuscrito. Ao Sistema Meteorológico do Paraná pelo fornecimento das planilhas metereológicas.

\section{REFERÊNCIAS BIBLIOGRÁFICAS}

ALMiRón, W.R. \& M.E. BREWER. 1996. Classification of immature stage habitat of Culicidae (Diptera) collected in Córdoba, Argentina. Mem. Inst. Oswaldo Cruz 91 (1): 1-9.

BAUMGARTNER, D.L. 1988. Suburban accumulations of discarded tires in northeastern Illinois and their associated mosquitoes. Jour. Amer. Mosq. Control Assoc. 4 (4): 500-507.

Beier, J.C.; M. Travis; C. Patricoski \& J. Krazfelder. 1983. Habitat segregation among larval mosquitoes (Diptera: Culicidae) in tire yards in Indiana, USA. Jour. Med. Entomol. 20 (1): 76-80.

BERRY, R.L.; E.D. PETERSON \& R. RESTIFO. 1988. Records of imported tire-breeding mosquitoes in Ohio. Jour. Amer. Mosq. Control Assoc. 4 (2): 187-189.

CHAHAD, S. \& A.L. Lozover. 1994. Distribuição sazonal de mosquitos imaturos (Diptera: Culicidae) em floreiras do cemitério municipal da Água Verde, Curitiba, Paraná, Brasil. Arq. Biol. Tecnol., Curitiba, 37 (4): 827-842.

EASTON, E.R. 1994. Urbanization and its effects on the ecology of mosquitoes in Macau, Southeast Asia. Jour. Amer. Mosq. Control Assoc. 10 (4): 540-544.

ForATtinI, O.P. 1962. Entomologia Médica. São Paulo, EDUSP, Vol. 1, 662p.

1998. Mosquitos Culicidae como vetores emergentes de infecções. Rev. Saúde Públ., São Paulo, 32 (6): 497-502.

ForATtinI, O.P.; I. KAKITANI; G.R.A.M. MARQUES \& M. BRITO. 1998a. Formas imaturas de anofelíneos em recipientes artificiais. Rev. Saúde Públ., São Paulo, 32 (2): 517-518.

1998b. Novos encontros de anofelíneos em recipientes artificiais. Rev. Saúde Públ., São Paulo, 32 (6): 598-599.

Forattini, O.P.; I. Kakitani \& M.A. Sallum. 1997. Encontro de criadouros de Aedes scapularis (Diptera: Culicidae) em recipientes artificiais. Rev. Saúde Públ., São Paulo, 31 (5): 519-22.

Revta bras. Zool. 19 (Supl. 1): $149-155,2002$ 
GETTMAN, A.D. \& D.W. HALL. 1989. A modification of scrap automobile tires for field studies of artificial container-breeding mosquitoes. Jour. Amer. Mosq. Control Assoc. 5 (3): 439.

LOPES, J. 1996. Ecologia de mosquitos (Diptera: Culicidae) em criadouros naturais e artificiais de área rural no Norte do Estado do Paraná, Brasil. IV. Espécies silvestres reproduzindo-se em recipientes. Arq. Biol. Tecnol., Curitiba, 39 (3): 671-676.

1997. Ecologia de mosquitos (Diptera: Culicidae) em criadouros naturais e artificiais de área rural no Norte do Estado do Paraná, Brasil. V. Coleta de larvas em recipientes artificiais instalados em mata ciliar. Rev. Saúde Públ., São Paulo, 31 (4): 370-377.

LoPES, J. \& A.L. Lozover. 1995. Ecologia de mosquitos (Diptera: Culicidae) em criadouros naturais e artificiais de área rural do Norte do Estado do Paraná, Brasil. I. Coletas ao longo do leito de ribeirão. Rev. Saúde Públ., São Paulo, 29 (3): 183-191.

Lopes, J.; M.A.N. Silva; A.M. Borsato; V.D.R.B. Oliveira \& F.J.A. Oliveira. 1993. Aedes (Stegomyia) aegypti L. e a culicideofauna associada em área urbana da região sul, Brasil. Rev. Saúde Públ., São Paulo, 27 (5): 326-333.

NASCI, R.S. 1988. Biology of Aedes triseriatus (Diptera: Culicidae) developing in tires in Louisiana. Jour. Med. Entomol. 25 (5): 402-405.

Silva, A.M. \& R.M.T. MENEZEs. 1996. Encontro de Aedes scapularis (Diptera: Culicidae) em criadouro artificial em localidade da região Sul do Brasil. Rev. Saúde Públ., São Paulo, 30 (1): 103-104.

Silva, M.A.N. \& A.L. Lozover. 1996. Criadouros de imaturos de mosquitos (Diptera, Culicidae) introduzidos em mata preservada na área urbana de Curitiba, Paraná, Brasil. Revta bras. Zool. 13 (4): 1023-1042.

ZAR, J.H. 1984. Biostatistical analysis. New Jersey, Prentice-Hall, 718p.

Recebido em 14.XI.2000; aceito em 13.VI.2002. 